\title{
Can electron multipacting explain the pressure rise in a cold bore superconducting undulator?
}

\author{
S. Casalbuoni, ${ }^{*}$ S. Schleede, D. Saez de Jauregui, M. Hagelstein, and P. F. Tavares ${ }^{\dagger}$ \\ Karlsruhe Institute of Technology, Institute of Synchrotron Radiation, P.O. Box 3640, D-76021 Karlsruhe, Germany
}

(Received 22 January 2010; published 7 July 2010)

\begin{abstract}
Preliminary studies performed with the cold bore superconducting undulator installed in the ANKA (Angstrom source Karlsruhe) storage ring suggest that the beam heat load is mainly due to the electron wall bombardment. Electron bombardment can both heat the cold vacuum chamber and induce an increase in the pressure because of gas desorption. In this contribution we compare the measurements of the pressure in a cold bore performed in the electron storage ring ANKA with the predictions obtained using the equations of gas dynamic balance in a cold vacuum chamber exposed to synchrotron radiation and electron bombardment. The balance results from two competing effects: the photon and electron stimulated desorption of the gas contained in the surface layer of the chamber wall and of the gas cryosorbed, and the cryopumping by the cold surface. We show that photodesorption alone cannot explain the experimental results and that electron multipacting is needed to reproduce the observed pressure rise. Electron bombardment can at the same time explain the observed beam heat load.
\end{abstract}

DOI: 10.1103/PhysRevSTAB.13.073201

PACS numbers: 29.27.- a, 41.75.Ht, 84.71.Ba

\section{INTRODUCTION}

In order to produce synchrotron radiation of highest brilliance, third generation synchrotron sources make use of insertion devices (IDs). The state of the art available today for IDs is the permanent magnet technology with magnet blocks placed inside the vacuum of the storage ring. Following an initial proposal at SPRING8 [1], the concept of cryogenic permanent magnet undulators (CPMU) is presently considered as a possible future evolution of in-vacuum undulators [2-5]. Superconducting undulators can reach, for the same gap and period length, higher fields even with respect to CPMU devices, allowing to increase the spectral range and the brilliance. At ANKA (Angstrom source Karlsruhe) we are running a research and development program on superconducting insertion devices (SCIDs). One of the key issues for the development of SCIDs is the understanding of the beam heat load to the cold vacuum chamber. The beam heat load is a fundamental input parameter for the design of SCIDs since it is needed to specify the cooling power.

Studies performed on the cold bore superconducting undulator installed at ANKA indicate that a simple model of electron bombardment could explain the beam heat load and observed pressure rise during normal user operation [6]. In this paper we go a step further solving the equations of gas dynamic balance in a cold vacuum chamber exposed to synchrotron radiation and electron bombardment. We show that the observed pressure rise can be explained by the occurrence of electron multipacting and not by photodesorption alone. The paper is organized as follows. For

\footnotetext{
*Corresponding author. sara.casalbuoni@kit.edu

On leave from ABTLuS/Brazilian Synchrotron Radiation Laboratory, Campinas, SP, Brazil.
}

completeness, in Secs. II and III we summarize, respectively, the experimental setup and the observations described in more detail in Ref. [6]. In Sec. IV we present the equations of gas dynamic balance and the input parameters derived from the literature and used to solve the model, whereas in Sec. V we derive an approximate analytical solution to those equations and discuss its properties. In Sec. VI we discuss the main results of the comparison between observations and simulations, and in Sec. VII we give some conclusions and outlook.

\section{EXPERIMENTAL SETUP}

ANKA is an electron storage ring used as a synchrotron facility [7]. A cold bore superconducting undulator built by ACCEL Instruments GmbH, Bergisch Gladbach, Germany [8], is installed in one of the four straight sections of the ring; the rest of the ring is at room temperature. The vacuum chambers of the warm part of ANKA have been baked before installation at $200^{\circ} \mathrm{C}$ for 48 hours and vented with nitrogen.

The storage ring compatible cryostat is shown in Fig. 1. The system is cryogen free and is cooled by three Sumitomo cryocoolers (RDK-408D @ $50 \mathrm{~Hz}$ ) [9]: two of them cool the coils to about $4 \mathrm{~K}$ and one cools the ultrahigh vacuum (UHV) tank, which is at $10 \mathrm{~K}$ and protects the coils from the external thermal radiation. The cryostat consists of two separate vacuum systems for the cold mass: an UHV vacuum system for the beam and an insulation vacuum system for the coils and the rest of the cold mass. The pressure of the two vacua are monitored by pressure gauges at room temperature. A $300 \mu \mathrm{m}$ stainless steel foil coated with $30 \mu \mathrm{m}$ of copper is placed between the cold mass and the beam vacuum. A taper system connects the normal beam pipe with the cold mass and has two functions: 


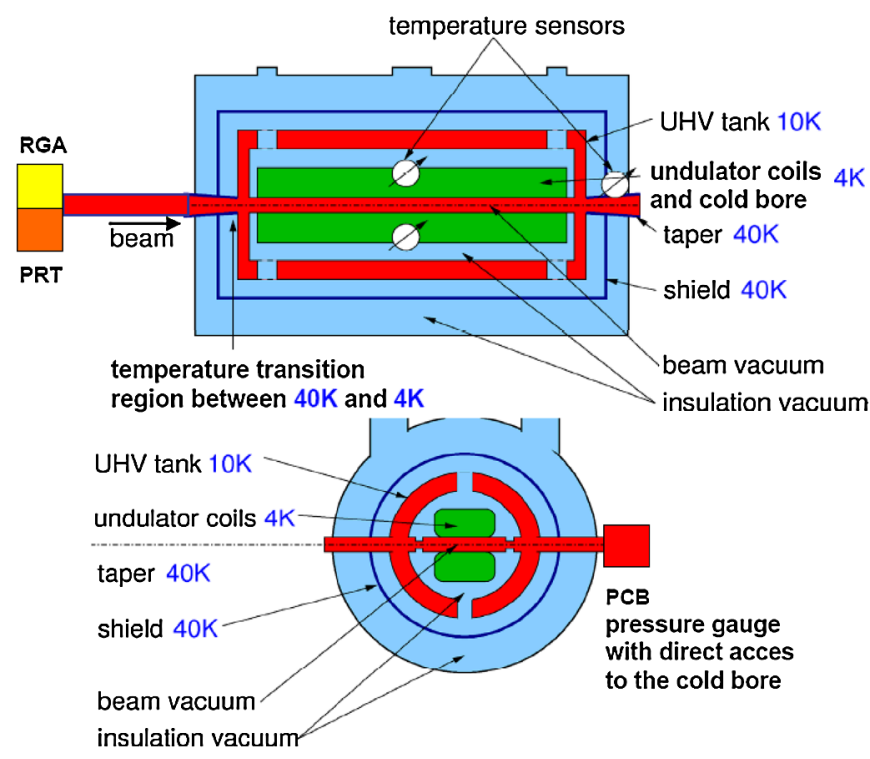

FIG. 1. (Color) Schematic layout of the vacuum system of the superconducting undulator and the position of the temperature sensors, a pressure gauge (PRT) and a residual gas analyzer (RGA) located in the room temperature region, and a pressure gauge with direct access to the cold bore (PCB).

(1) smooth transition for wake fields; (2) thermal transition between the cold bore at $4 \mathrm{~K}$ and the beam pipe at room temperature. Several temperature sensors are placed on the different elements: coils, UHV tank, taper entrance, taper exit, etc. A pressure gauge (PRT) and a residual gas analyzer (RGA) are located in the room temperature region about $0.5 \mathrm{~m}$ upstream from the entrance of the undulator cryostat. A unique diagnostic in this undulator, absent in other cold bore wigglers installed in the different synchrotron light sources $[10,11]$ (because of the different design), is a pressure gauge with direct access to the cold bore (PCB). The undulator vacuum chamber at $4.2 \mathrm{~K}$ is $1.4 \mathrm{~m}$ long, it has a rectangular cross section with $66 \mathrm{~mm}$ width. The undulator can be operated with different gap sizes: 16, 12 , and $8 \mathrm{~mm}$, and it can be opened to $29 \mathrm{~mm}$ without current in the coils during injection. The height of the beam vacuum chamber changes accordingly. In this paper we describe results obtained with the beam stay clear height of $29 \mathrm{~mm}$ and no current in the coils, which means no magnetic field. In order to protect the undulator from the synchrotron radiation emitted by the upstream magnets, a collimator system is located at about $1 \mathrm{~m}$ from the entry point of the undulator [12].

\section{OBSERVATIONS}

The superconducting undulator has been operating in the ANKA storage ring since 2005 [8]. The beam heat load and the pressure in the cold vacuum chamber have been monitored since then. A typical run is shown in Fig. 2 where the average beam current, the beam energy, the UHV pressure [13], and the temperature of the coils are reported as a

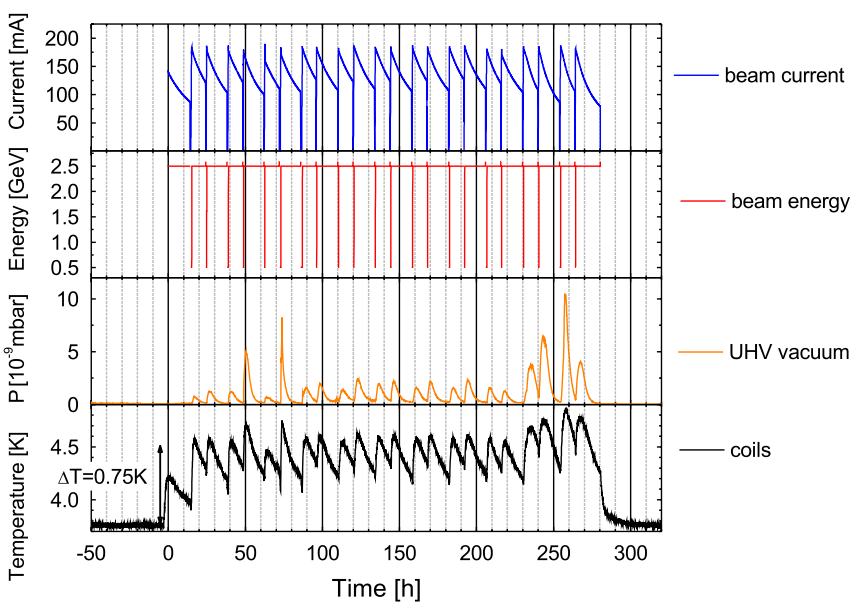

FIG. 2. (Color) Typical user operation run with open gap $(=29 \mathrm{~mm})$ and no current in the undulator. The beam current, the beam energy, the UHV pressure, and the temperature of the coils are reported as a function of time.

function of time. The temperature increase of the coils can be converted into the deposited beam heat load. The calibration has been performed using a resistor in thermal contact with the coils. The time constant to reach thermal equilibrium is of the order of 2 hours. The beam heat load to the coils is about $1 \mathrm{~W}$.

A pressure rise is observed after beam injection. A correlation between the heat load and the pressure is observed in several runs, see Fig. 3. In Fig. 4 a comparison of the pressure behavior in the cold bore (green squares) and in the room temperature region (red triangles) is displayed. In the cold bore the pressure reaches a maximum after 1-3 hours from injection, while in the room temperature region this happens within a few minutes. The plot shows also that the decay of the pressure in the cold bore is much faster than the decay of the pressure in the room temperature region. The dynamic pressure increases nonlinearly with the average beam current [6]. A similar pressure rise with current has been observed in positron rings (machines at room temperature) and has been attrib-

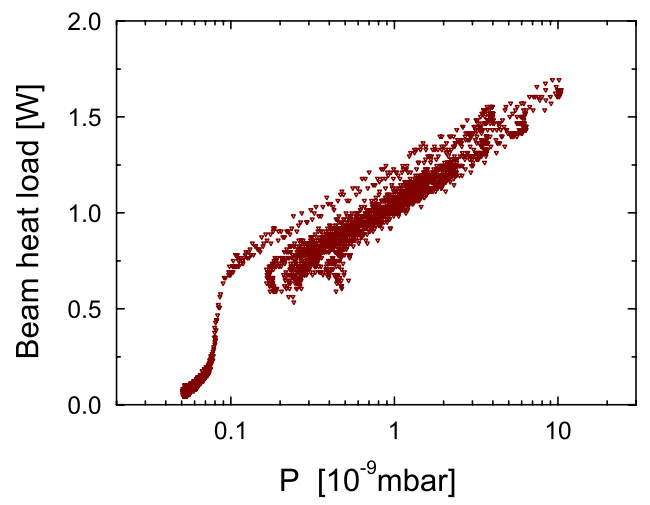

FIG. 3. (Color) The beam heat load as a function of the UHV pressure in the cold bore from Fig. 2. 


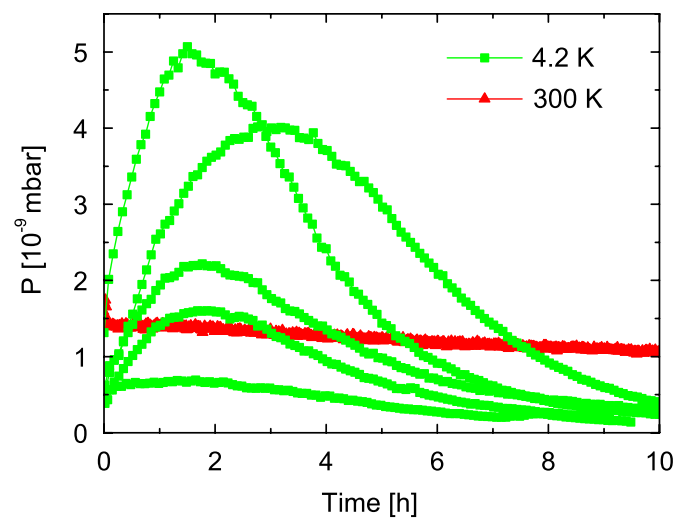

FIG. 4. (Color) Comparison of the dynamic pressure in the cold bore (green squares) with the one in the room temperature region (red triangles). The static pressure in the cold bore (PCB) is about $2 \times 10^{-11} \mathrm{mbar}$ and in the room temperature region (PRT) is about $2 \times 10^{-10} \mathrm{mbar}$.

uted to electron multipacting $[14,15]$. We will come back to this in Sec. VI.

The mass spectrum (RGA) of the warm vacuum chamber with beam shows, while the undulator is cold, only the $\mathrm{H}_{2}$ and $\mathrm{CO}$ lines, see Fig. 5. $\mathrm{CO}$ disappears when there is no beam. In the rest of the ring most of the time no $\mathrm{CO}$ is detected. The mass spectrum measured by warming up the undulator to room temperature in absence of electron beam shows together with $\mathrm{H}_{2}$ the presence of $\mathrm{CO}, \mathrm{CO}_{2}$, and $\mathrm{H}_{2} \mathrm{O}$, indicating that the cryosorbed gas layer might have a more complex gas composition than simply $\mathrm{H}_{2}$. However, $\mathrm{H}_{2}$ is the only gas among the ones mentioned above that has a non-negligible vapor pressure at $4-20 \mathrm{~K}$ and we see that this is the main gas component measured when the undulator is cold.

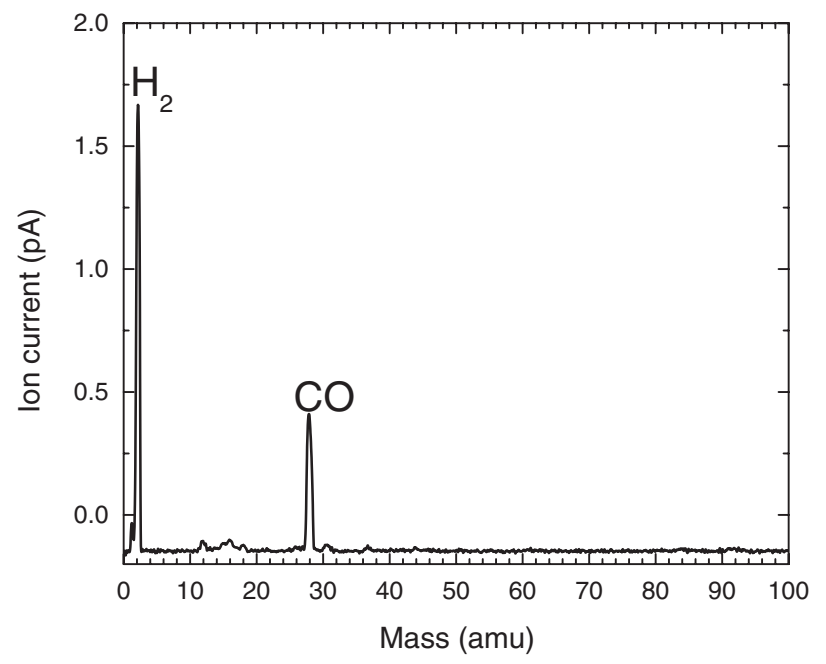

FIG. 5. Mass spectrum of the warm vacuum chamber just before the undulator with beam measured with the RGA indicated in Fig. 1.

\section{MODEL AND INPUT PARAMETERS}

The equations of gas dynamic balance inside a vacuum chamber can be written as (see Refs. $[16,17]$ and references therein)

$$
\begin{aligned}
& V \frac{d n}{d t}=q+q^{\prime}(s)-\alpha S\left[n-n_{e}(s, T)\right]+u \frac{d^{2} n}{d z^{2}}, \\
& A \frac{d s}{d t}=\alpha S\left[n-n_{e}(s, T)\right]-q^{\prime}(s),
\end{aligned}
$$

where $n$ is the volume gas density, $s$ the surface density of the cryosorbed gas, $V$ the vacuum chamber volume, $A$ the vacuum chamber wall area, $q$ is the primary beam induced desorption flux, $q^{\prime}$ the secondary beam induced desorption flux (desorption of cryosorbed molecules), $\alpha$ the sticking coefficient, $S=A \bar{\nu} / 4$ is the ideal wall pumping speed, $\bar{\nu}$ is the mean molecular speed, $n_{e}$ the thermal equilibrium gas density, and $u$ the specific vacuum chamber conductance per unit axial length. In the following, we consider the gas to consist only of $\mathrm{H}_{2}$.

The specific vacuum chamber conductance per unit axial length is given by $u=A_{c} D$, where $D=2 A_{c} \bar{\nu} / 3$ is the Knudsen diffusion coefficient and $A_{c}$ the area of the rectangular cross section of the vacuum chamber. Axial diffusion can be neglected when $D A_{c} / L^{2} \ll S \alpha$ [16], which means

$$
\frac{8}{3} \frac{A_{c}^{2}}{A L^{2}} \ll \alpha
$$

Even for the lowest experimental value of the sticking coefficient for $\mathrm{H}_{2}$ at $4.2 \mathrm{~K}, \alpha=0.02$ [18] condition (2) is satisfied for the geometry of the undulator vacuum chamber where $L=1.4 \mathrm{~m}$ and for a gap of $29 \mathrm{~mm}, A_{c}=$ $0.00191 \mathrm{~m}^{2}$ and $A=0.266 \mathrm{~m}^{2}$. Therefore in the following we neglect axial diffusion $u d^{2} n / d z^{2} \approx 0$.

The beam induced desorption flux consists of photon (PSD) and electron (ESD) stimulated desorption:

$$
q=\eta \dot{\Gamma}+\phi \dot{\Theta}, \quad q^{\prime}=\eta^{\prime} \dot{\Gamma}+\phi^{\prime} \dot{\Theta},
$$

where $\eta$ and $\eta^{\prime}$ are the primary and secondary electron stimulated desorption yields, $\dot{\Gamma}$ is the electron flux, $\phi$ and $\phi^{\prime}$ are the primary and secondary photodesorption yields, and $\dot{\Theta}$ is the photon flux. The photon flux is proportional to the beam current, so we consider it to decay exponentially with time as $\dot{\Theta}=\dot{\Theta}_{0} \exp (-t / \tau)$, where $\tau=80000 \mathrm{~s}$ is the beam lifetime which is about 22 hours. For the ANKA cold bore vacuum chamber with gap $=29 \mathrm{~mm}$ and average beam current $I=150 \mathrm{~mA}$, the photon flux impinging on the lower and upper surfaces is $\dot{\Theta}_{0}=$ $5 \times 10^{15}$ photons/s. The photon flux $\dot{\Theta}_{0}$ is obtained by integrating the angular and spectral distribution of number of photons emitted by the upstream dipole over all photon energies and over the horizontal and vertical acceptance defined by the cold bore geometry and distance to the 
upstream bending magnet. We assume that the electron flux also decays exponentially in time: $\dot{\Gamma}=$ $\dot{\Gamma}_{0} \exp \left(-t / \tau_{\mathrm{el}}\right)$. In order to estimate $\dot{\Gamma}_{0}$, we assume that the bombarding electrons are initially generated (e.g. by photoionization of the cryosorbed $\mathrm{H}_{2}$ molecules on the cold surface) at rest, and then accelerated against the wall by the transverse electric field of the electron bunch. For a typical $3.6 \times 10^{9}$ electrons/bunch, we obtain a mean electron energy $\Delta W=10 \mathrm{eV}$ [19] so that if the observed beam heat load $P=1 \mathrm{~W}$ is to be explained by electron bombardment, then $\dot{\Gamma}_{0}=6 \times 10^{17}$ electrons $/ \mathrm{s}$.

The measurements of input parameters such as the photon and electron primary and secondary desorption yields, as well as the sticking coefficient, are quite challenging. Several experiments have been performed to measure those parameters for a $\mathrm{H}_{2}$ layer cryosorbed on a copper substrate at low temperatures and a wide range of values can be found in the literature. The photon and electron primary and secondary desorption yields, as well as the sticking coefficient depend on the temperature, on the surface coverage, on the geometry (closed or open), on the photon, and on the electron energy distribution and dose. The different experiments reported in the literature have been performed under a variety of conditions, and it is therefore difficult to compare them with each other and to extract the values needed for a consistent comparison with our experimental situation. Even though a comprehensive review of different experimental results on the above-mentioned parameters is beyond the scope of this paper, we list below some of the values obtained in experiments performed in the past 20 years to understand the beam vacuum system of a cold bore accelerator, which started with studies motivated by the $20 \mathrm{TeV}$ Superconducting Super Collider [20] and continued with studies motivated by the Large Hadron Collider (LHC) [21].

The photon primary desorption yield $\phi$ and the ratio of the secondary photodesorption yield $\phi^{\prime}$ to the sticking coefficient $\alpha$ have been measured on a copper electroplated stainless steel liner at $4.2 \mathrm{~K}$ in a quasiclosed geometry by Anashin et al. [22] to vary in the range $2 \times 10^{-4} \leq \phi \leq 5 \times 10^{-2}$ and $5 \times 10^{-2} \leq \phi^{\prime} / \alpha \leq 8$.

In a more recent work [23], measurements of the sticking coefficient $\alpha$ and of the sum of the primary and secondary electron stimulated desorption yields have been reported. The sticking coefficient ranges $0.25 \leq \alpha \leq$ 0.6 for surface coverages of about one monolayer $s_{m}=$ $3 \times 10^{19}$ molecules $/ \mathrm{m}^{2}$. The sum of the primary and secondary electron stimulated desorption yield for $300 \mathrm{eV}$ electrons has been measured as a function of $\mathrm{H}_{2}$ coverage at about $2 \mathrm{~K}$ on the LHC beam screen to be $50 \leq \eta+\eta^{\prime} \leq$ 2000.

The ratio of the sum of the primary and recycling electron stimulated desorption yield to the sticking coefficient can be estimated from our measurements [6]. Following Ref. [24], we use the equation

$$
\frac{q+q^{\prime}}{\alpha}=S\left[n-n_{e}(s, T)\right]=S G \Delta P,
$$

where $\Delta P=P_{\max }-P_{e}$ with $P_{e} \lesssim 2 \times 10^{-11}$ mbar, the thermal equilibrium pressure at $4.2 \mathrm{~K}$, and

$$
G=\frac{1}{k_{B} \sqrt{T T_{\mathrm{RT}}}}=2 \times 10^{23} \mathrm{~m}^{-3} \mathrm{mbar}^{-1}
$$

with $T=4.2 \mathrm{~K}$ and $T_{R T}=300 \mathrm{~K}$. As mentioned above, the photon flux on the ANKA cold bore vacuum chamber with $\mathrm{gap}=29 \mathrm{~mm}$ and $I=150 \mathrm{~mA}, \quad \dot{\Theta}_{0}=5 \times$ $10^{15}$ photons $/ \mathrm{s}$, and the estimated electron flux to explain a heat load of $P=1 \mathrm{~W}$ (for $10 \mathrm{eV}$ electrons) is $\dot{\Gamma} \approx 6 \times$ $10^{17}$ electrons/s. Being $\phi+\phi^{\prime} \lesssim \eta+\eta^{\prime}[24,25]$, we can neglect the contribution of PSD to the beam desorption flux, so that $q=\eta \dot{\Gamma}$ and $q^{\prime}=\eta^{\prime} \dot{\Gamma}$. The observed $\Delta P$ ranges from $2 \times 10^{-11}$ mbar to $8 \times 10^{-8}$ mbar [6]. For $\mathrm{H}_{2}$, the mean molecular speed at $4.2 \mathrm{~K}$ is $\bar{\nu}=210 \mathrm{~m} / \mathrm{s}$ and applying Eq. (4) we find that the sum of the primary and secondary desorption yields $\left(\eta+\eta^{\prime}\right) / \alpha$ for $\mathrm{H}_{2}$ ranges between $10^{-4}$ to 4 molecules/electron. Our values are in good agreement with the ones measured at COLDEX [24] that range between $10^{-2}$ molecules/electron for an electron dose of $2 \times 10^{23}$ electrons $/ \mathrm{m}^{2}$ to 30 molecules/electron for an electron dose of $10^{21}$ electrons $/ \mathrm{m}^{2}$, considering that in our case the temperature is lower ( $4.2 \mathrm{~K}$ instead of $12 \mathrm{~K}$ ), the mean electron energy is an order of magnitude smaller $(10 \mathrm{eV}$ instead of $100 \mathrm{eV}$ [24]) and that our electron dose is in some cases much higher (after two weeks of normal user operation it is about $2 \times 10^{24}$ electrons $/ \mathrm{m}^{2}$ ).

In Ref. [22] it has been shown that the secondary photodesorption yield $\phi^{\prime}$ depends linearly on the surface coverage up to one monolayer $\phi^{\prime}=\phi_{0}^{\prime}\left(s / s_{n}\right)$. Similar results have been found by Tratnik [23] for the sum of the primary and secondary electrodesorption yields $\eta+\eta^{\prime}$ up to one monolayer. Considering the results obtained in Ref. [24], being $5 \times 10^{-4} \leq \eta \leq 10^{-1}$, to solve Eqs. (1) we assume $\eta^{\prime}=\eta_{0}^{\prime}\left(s / s_{n}\right)$. In our model we define the normalization of the surface coverage to be $s_{n}=10^{18}$ molecules $/ \mathrm{m}^{2}$.

The volume gas density $n$ at a temperature $T$ is related to the pressure measured at room temperature by

$$
n=\frac{P}{k_{B} \sqrt{T T_{\mathrm{RT}}}} .
$$

The value of the volume gas density at $t=0$ (injection time) $n_{0}$ is obtained from Eq. (5) with $P=P_{0}$ [mbar] chosen to fit the experimental data. We have assumed $n_{e}(s, T)=4 \times 10^{12}$ molecules $/ \mathrm{m}^{3}$, corresponding to an equilibrium pressure $P_{e}=2 \times 10^{-11}$ mbar. The surface coverage at equilibrium is constrained to be $s_{0}<1.5 \times$ $10^{19}$ molecules $/ \mathrm{m}^{2}$ by the measured adsorption isotherms of $\mathrm{H}_{2}$ on copper plated stainless steel at $4.2 \mathrm{~K}$ from Ref. [26]. 


\section{APPROXIMATE ANALYTICAL SOLUTIONS TO THE GAS DYNAMIC BALANCE EQUATIONS}

Before embarking on a direct numerical computation of the solutions to Eqs. (1), we obtain a closed expression of the solution for a simplified form of these equations, namely, when only photodesorption is present (i.e., assuming zero electron flux). Apart from providing a cross-check on the full numerical calculations described in Sec. VI below, this simplified situation is interesting because, as our analysis will show, it cannot reproduce the experimental data, indicating that the additional ingredient of electron bombardment (and multipacting) is indeed essential. In fact, this approach allows us to obtain several relevant properties of the solutions, as well as an approximate analytical solution, valid in the limit of times short compared to the beam lifetime, which allows us to set bounds on the characteristic time constants associated with the pressure rise when only photons are present.

When the electron flux is zero, Eqs. (1) reduce to

$V \frac{d n}{d t}=\frac{1}{\tau_{\epsilon}} e^{-t / \tau}+\frac{A}{\tau_{d}} e^{-t / \tau} s-\alpha S\left(n-n_{e}\right)$,

$A \frac{d s}{d t}=\alpha S\left(n-n_{e}\right)-\frac{A}{\tau_{d}} e^{-t / \tau} s$,

where we have defined the time constants

$$
\tau_{\epsilon}=\frac{1}{\phi \dot{\Theta}_{0}} \quad \tau_{d}=\frac{A s_{n}}{\phi_{0}^{\prime} \dot{\Theta}_{0}} .
$$

The total number of molecules (on the surface + in the volume)

$$
N_{T}(t)=V n(t)+A s(t)
$$

satisfies the equation

$$
\frac{d N_{T}}{d t}=q=\frac{1}{\tau_{\epsilon}} e^{-t / \tau},
$$

with the trivial solution

$$
N_{T}(t)=\frac{\tau}{\tau_{\epsilon}}\left(1-e^{-t / \tau}\right)+N_{T 0} \quad N_{T 0}=V n_{0}+A s_{0} .
$$

Writing

$$
s(t)=\frac{N_{T}(t)-V n(t)}{A},
$$

we can now decouple the two equations and write an equation involving $n(t)$ only:

$$
\frac{d n}{d t}+g(t) n(t)=k(t)
$$

where

$$
\begin{aligned}
& g(t)=\frac{e^{-t / \tau}}{\tau_{d}}+\frac{1}{\tau_{s}} \\
& k(t)=\frac{e^{-t / \tau}}{V \tau_{\epsilon}}+\frac{n_{e}}{\tau_{s}}+\frac{1}{V \tau_{d}} e^{-t / \tau}\left[\frac{\tau}{\tau_{\epsilon}}\left(1-e^{-t / \tau}\right)+N_{T 0}\right],
\end{aligned}
$$

with

$$
\tau_{s}=\frac{V}{\alpha S} .
$$

This can be solved by writing

$$
\begin{gathered}
g(t)=\frac{1}{h(t)} \frac{d h}{d t} \quad \frac{1}{h} \frac{d(n h)}{d t}=\frac{d n}{d t}+g(t) n(t)=k(t) \\
h(t) n(t)-h_{0} n_{0}=\int_{0}^{t} h\left(t^{\prime}\right) k\left(t^{\prime}\right) d t^{\prime}
\end{gathered}
$$

and finally

$$
n(t)=\frac{n_{0} h_{0}}{h(t)}+\frac{1}{h(t)} \int_{0}^{t} h\left(t^{\prime}\right) k\left(t^{\prime}\right) d t^{\prime},
$$

with

$$
h(t)=\exp \left[\frac{\tau}{\tau_{d}}\left(1-e^{-t / \tau}\right)+\frac{t}{\tau_{s}}\right] \quad h_{0}=1 .
$$

Equation (14) gives the molecular density (and therefore the pressure) as a function of time in the form of a simple integral. Even without solving this integral, we may obtain the limiting behavior of the solutions for very large time

$$
\begin{gathered}
N_{T}(t \rightarrow \infty) \rightarrow \frac{\tau}{\tau_{\epsilon}}+N_{T 0} \quad n(t \rightarrow \infty) \rightarrow n_{e} \\
s(t \rightarrow \infty) \rightarrow \frac{N_{T 0}-V n_{e}}{A}+\frac{\tau}{\tau_{e} A} .
\end{gathered}
$$

Finally, in the limit $\tau \rightarrow \infty$, we obtain the approximate solution

$$
\begin{aligned}
n(t)= & n_{0} e^{-t / \tau_{d s}}+\tau_{d s}\left(\frac{N_{T 0}}{V \tau_{d}}+\frac{1}{V \tau_{\epsilon}}+\frac{n_{e}}{\tau_{s}}\right)\left(1-e^{-t / \tau_{d s}}\right) \\
& +\frac{\tau_{d s}^{2}}{V \tau_{d} \tau_{\epsilon}}\left(\frac{t}{\tau_{d s}}-1+e^{-t / \tau_{d s}}\right) .
\end{aligned}
$$

where we have defined yet another time constant

$$
\tau_{d s}=\frac{1}{\frac{1}{\tau_{d}}+\frac{1}{\tau_{s}}} .
$$

Noting that, for typical parameters, $\tau_{d s} \ll \tau$, we see from Eq. (16) that, for times such that $\tau_{d s} \ll t \ll \tau$, the volume density and therefore the pressure grows linearly with time. Clearly, this approximation cannot give us the exact time at which the pressure reaches its maximum value, but it does indicate that such a maximum cannot happen at times much shorter than the beam lifetime $\tau$ since for that time range the molecular density is a monotonically increasing function of time. However, this is precisely what the experimental data show, since the life- 
time is typically of the order of 20 hours, whereas the pressure peak happens within just a few hours. This leads us to assume that some other mechanism, apart from photodesorption and with a different characteristic time constants must be involved to explain the experimental observations. In the following section, we discuss this in more detail, using a full numerical solution to the gas dynamic balance equations.

\section{RESULTS}

Various simulations have been performed solving Eqs. (1). The idea is to change the input parameters within the range of values found in the literature and to compare the pressure simulated with the one measured.

As we have seen above, considering just the contribution of photons ( $\dot{\Gamma}=0$ electrons/s) to desorb molecules from the cold surface it is impossible to reproduce the measured values of the pressure as a function of time. This can also be demonstrated by showing that the first of Eqs. (1) at the time at which the pressure has a maximum $t_{\max }$ is not satisfied. Since at $t_{\max } d n / d t=0$ it follows

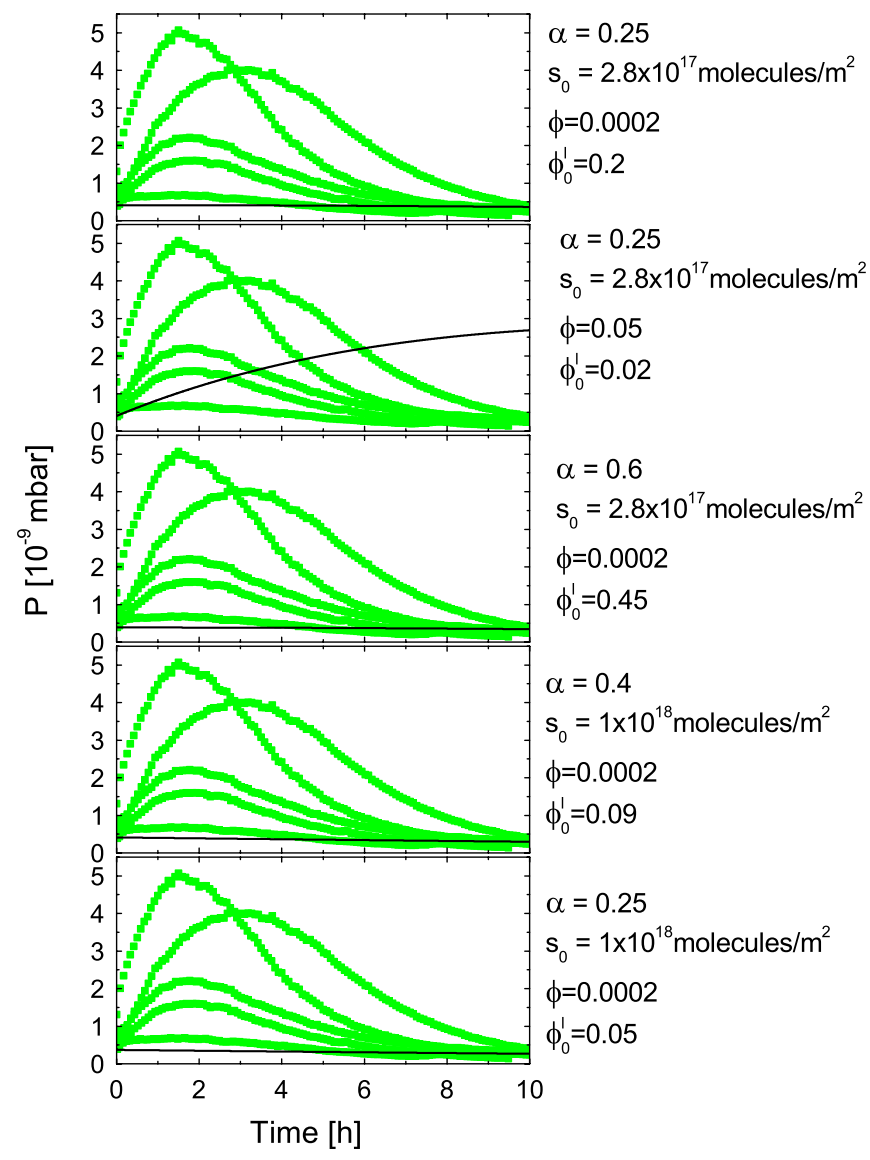

FIG. 6. (Color) Pressure in the cold vacuum chamber as a function of time. The green squares indicate the typical behavior and range of measured values. The black line displays the simulations shown in these plots consider only photodesorption ( $\dot{\Gamma}_{0}=0$ electrons $\left./ \mathrm{s}\right)$.

$$
\exp \left(-t_{\max } / \tau\right)=\frac{\alpha G S \Delta P}{\left(\phi+\phi^{\prime}\right) \dot{\Theta}_{0}}
$$

The experimental constraints on $2 \times 10^{-4} \leq \phi \leq$ $5 \times 10^{-2}, 5 \times 10^{-2} \leq \phi^{\prime} / \alpha \leq 8$ (Ref. [22]), and on $0.25 \leq \alpha \leq 0.6$ (Ref. [23]) described in the previous section limit $\left(\phi+\phi^{\prime}\right) / \alpha$ in the range $0.05 \leq\left(\phi+\phi^{\prime}\right) / \alpha \leq$ 8.2. With this in mind and recalling from the observations that $\Delta P \simeq 5 \times 10^{-9}$ mbar, the second term of Eq. (18) is always less than 0.02. From our experimental data $t_{\max } \simeq$ 2 hours and $\tau \simeq 22$ hours, so the first term of Eq. (18) is about 1 and Eq. (18) is not satisfied.

The solutions obtained from Eqs. (1) taking into account only photodesorption with different sets of parameters are reported in Fig. 6. The initial pressure is set to $P_{0}=4 \times$ $10^{-10}$ mbar. The results obtained considering also electron stimulated desorption are shown in Fig. 7. We have used the values indicated in the column "fixed" of Table I which fit one of the green curves, see Fig. 7. In order to study the

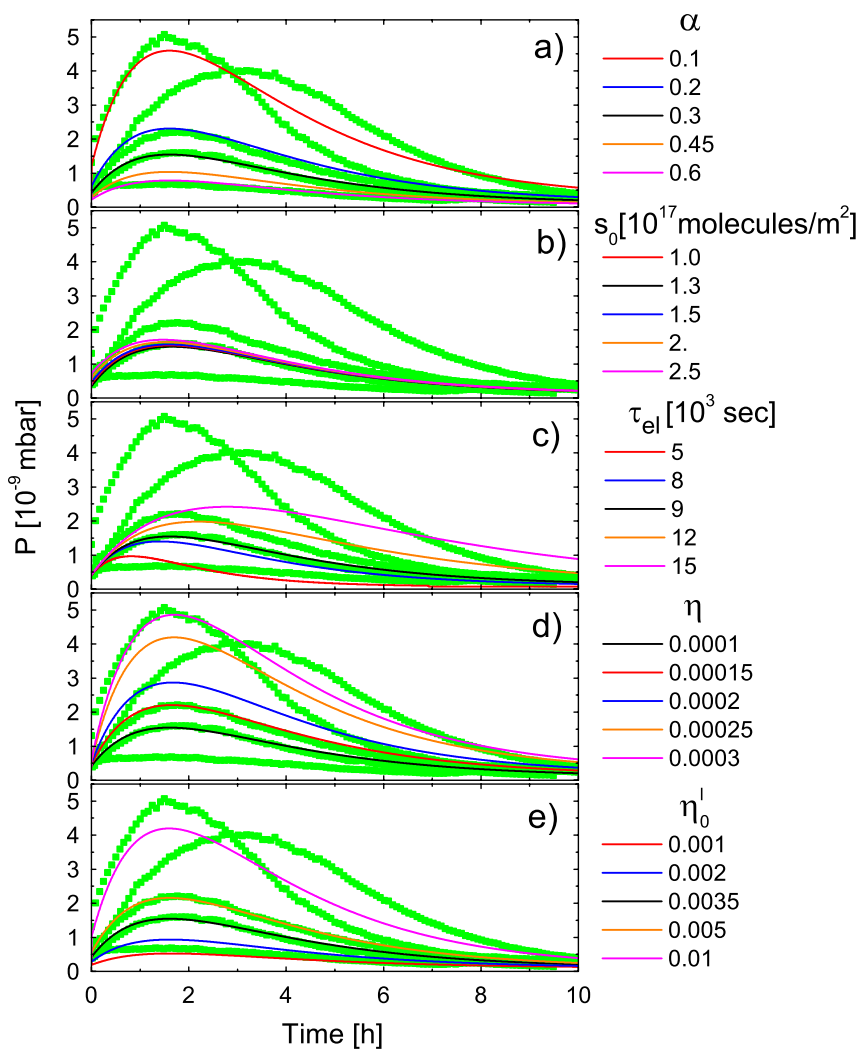

FIG. 7. (Color) Pressure in the cold vacuum chamber as a function of time. The green squares indicate the typical behavior and range of measured values. The simulations shown in these plots are obtained considering also electron stimulated desorption and using the "fixed" values of the input parameters as in Table I varying (a) the sticking coefficient $\alpha$, (b) the initial value of the $\mathrm{H}_{2}$ surface coverage $s_{0}$, (c) the decay time of the electrons desorbing $\mathrm{H}_{2}$ from the surface $\tau_{\mathrm{el}}$, (d) the primary electron stimulated desorption yield $\eta$, and (e) the secondary electron stimulated desorption yield $\eta^{\prime}$. 
TABLE I. Values used as input parameters in Eqs. (1) to obtain the values of the pressure as a function of time reported in Fig. 7.

\begin{tabular}{lccc}
\hline \hline & $\min$ & $\max$ & Fixed \\
\hline$s_{0}\left(10^{17}\right.$ molecules $\left./ \mathrm{m}^{2}\right)$ & 1 & 2.5 & 1.3 \\
$\alpha$ & 0.1 & 0.6 & 0.3 \\
$\phi$ & & & 0.0002 \\
$\phi_{0}^{\prime}$ & & & 0.01 \\
$\eta$ & 0.0001 & 0.0003 & 0.0001 \\
$\eta_{0}^{\prime}$ & 0.001 & 0.01 & 0.0035 \\
$\dot{\Theta}_{0}\left(10^{15}\right.$ photons/s) & & & 5 \\
$\dot{\Gamma}_{0}\left(10^{17}\right.$ electrons/s) & & & 6 \\
$\tau(\mathrm{s})$ & & & 80000 \\
$\tau_{\mathrm{el}}(\mathrm{s})$ & 5000 & 15000 & 9000 \\
\hline \hline
\end{tabular}

effect of the different parameters, we have performed different simulations by varying the parameters shown in Table I within the values indicated in the columns " $m i n$ " and "max". Increasing the sticking coefficient $\alpha$ or decreasing the primary and secondary electron stimulated desorption yield decreases the amount of molecules desorbed and the pressure peak while a change in the surface coverage $s_{0}$ does not significantly affect the results. Figure 7(c) also shows that an increase in the decay time of the impinging electrons $\tau_{\text {el }}$ delays the pressure peak. In Fig. 8 we show that it is possible to tune the input parameters within the range of values found in the literature to reproduce the different measured curves of the pressure in the cold bore. We conclude that, taking into account the contribution of molecules desorbed by electrons, it is possible to reproduce the observed behavior of the pressure by varying the input parameters in the range of values found in the literature. The measurements are well reproduced by using a decay time of the electrons desorbing $\mathrm{H}_{2}$ from the surface in the range $8000 \mathrm{~s}<\tau_{\mathrm{el}}<13000 \mathrm{~s}$. Since the beam current $I_{b}$ and the flux of electrons bombarding the wall $\dot{\Gamma}_{0}$ decay exponentially with time with two different time constants, respectively $\tau$ and $\tau_{\text {el }}$,

$$
I_{b}=I_{b 0} \exp (-t / \tau), \quad \dot{\Gamma}=\dot{\Gamma}_{0} \exp \left(-t / \tau_{\mathrm{el}}\right)
$$

where $I_{b 0}$ is $I_{b}$ at $t=0$, it follows that

$$
\dot{\Gamma}=\dot{\Gamma}_{0} \exp \left[\tau / \tau_{\mathrm{el}} \ln \left(I_{b} / I_{b 0}\right)\right]=\dot{\Gamma}_{0}\left(\frac{I_{b}}{I_{b 0}}\right)^{\tau / \tau_{\mathrm{el}}}
$$

An example of this power law dependence of the flux of the electrons bombarding the wall and desorbing $\mathrm{H}_{2}$ molecules as a function of the beam current is shown in Fig. 9. The behavior of the electron flux $\dot{\Gamma}$ as a function of the beam current $I_{b}$ displays a growth much faster than linear showing an avalanche effect, which has often been described in the literature as multipacting. The mechanism generating this drastic increase of electrons impinging the wall with beam current is still not clear.

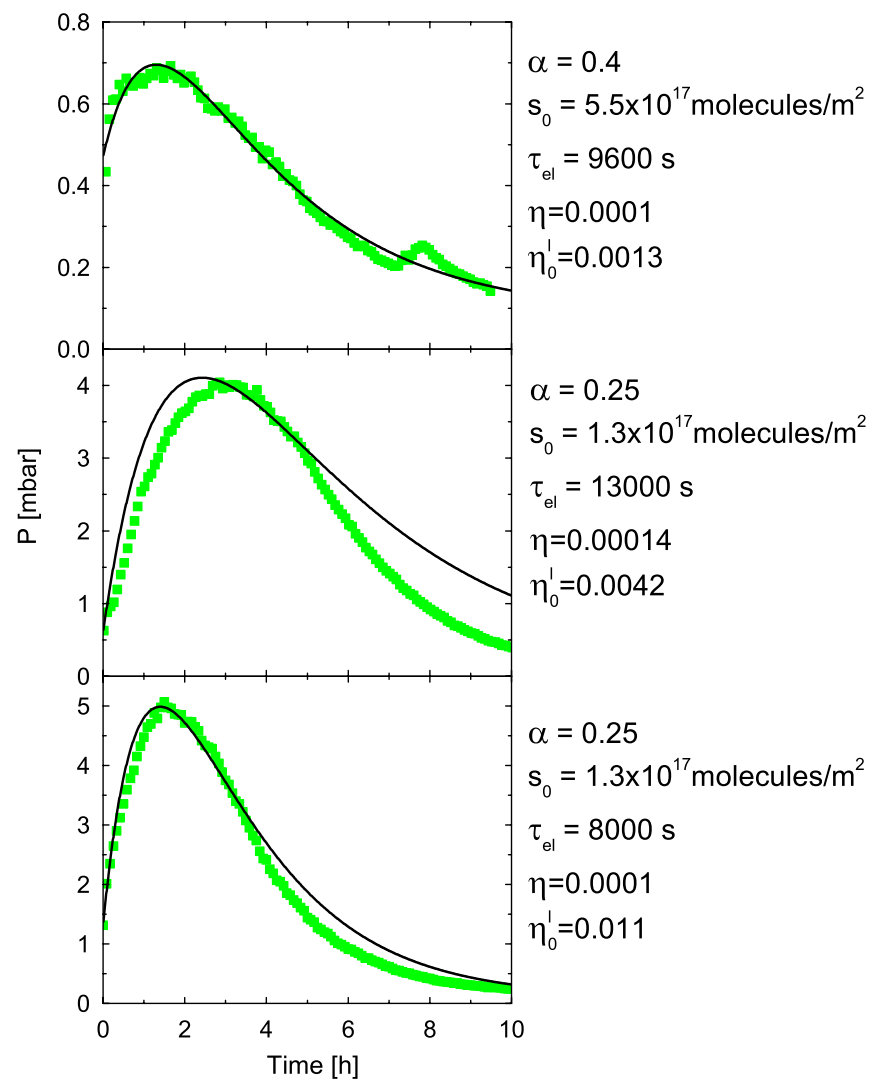

FIG. 8. (Color) Pressure in the cold vacuum chamber as a function of time. The green squares indicate the typical behavior and range of measured values. The simulations shown in these plots demonstrate that it is possible to tune the input parameters within the range of values found in the literature to reproduce the different measured curves of the pressure in the cold bore.

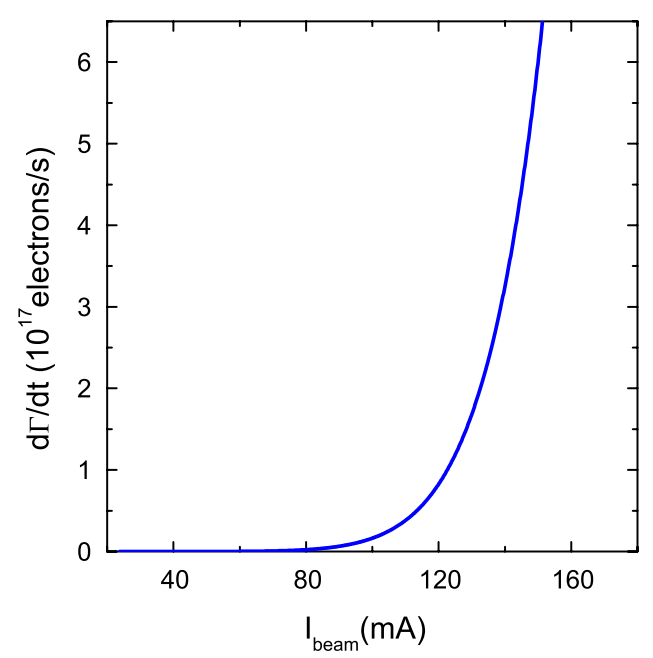

FIG. 9. (Color) Flux of the electrons desorbing $\mathrm{H}_{2}$ molecules from the surface as a function of the beam current for a decay time $\tau_{\mathrm{el}}=9000 \mathrm{~s}$. The behavior of the electron flux $\dot{\Gamma}$ as a function of the beam current $I_{b}$ displays a growth much faster than linear showing an avalanche effect, which has often been described in the literature as multipacting. 


\section{CONCLUSIONS AND OUTLOOK}

A simple model of electron bombardment appears to be consistent with the beam heat load and pressure rise observed in the cold bore of the superconducting undulator installed at ANKA. A common cause of electron bombardment is the buildup of an electron cloud, which strongly depends on the chamber surface properties. The surface properties as secondary electron yield, photoemission yield, photoemission induced electron energy distribution, needed in the simulation codes to determine the eventual occurrence and size of an electron cloud buildup, have only partly been measured for a cryosorbed gas layer. Even using uncommonly large values for these parameters, the heat load inferred from the ECLOUD simulations [27] is about 1 order of magnitude lower than the measurements [28]. Mechanisms different than the classical buildup of the electron cloud that can explain the electron bombardment and multipacting are currently under study.

We have shown that in order to reproduce the pressure measurements it is necessary to include electron stimulated desorption with a shorter decay time $\tau_{\mathrm{el}}$ than the beam lifetime $\tau$. This implies a very fast avalanchelike growth of the electron flux $\dot{\Gamma}$ as a function of beam current suggesting electron multipacting. Considering the simplified assumptions, for example, the gas made by $\mathrm{H}_{2}$ only and the large measurement uncertainties, the agreement between simulations and measurements is satisfying. A refinement of the model makes sense once more accurate and controlled measurements will be available with the planned cold vacuum chamber (COLDDIAG) to be installed in a storage ring, implemented with the following diagnostics: (i) retarding field analyzers to measure the electron flux; (ii) temperature sensors to measure the total heat load; (iii) pressure gauges; (iv) and mass spectrometers to measure the gas content [29].

\section{ACKNOWLEDGMENTS}

The authors are grateful to Frank Zimmermann (CERN) for useful discussions in the initial phase of this project. Pedro F. Tavares acknowledges financial support from the Brazilian Research Council-CNPq.

[1] T. Hara, T. Tanaka, H. Kitamura, T. Bizen, X. Marchal, T. Seike, T. Kohda, and Y. Matsuura, Phys. Rev. ST Accel. Beams 7, 050702 (2004).

[2] C. Kitegi, J. Chavanne, D. Cognie, P. Elleaume, F. Revol, C. Penel, B. Plan, and M. Rossat, in Proceedings of the 10th European Particle Accelerator Conference, Edinburgh, Scotland, 2006 (EPS-AG, Edinburgh, Scotland, 2006).

[3] T. Tanabe, D. A. Harder, G. Rakowsky, T. Shaftan, and J. Skaritka, in Proceedings of the 2007 Particle Accelerator Conference, Albuquerque, New Mexico (IEEE, Albuquerque, New Mexico, 2007).
[4] C. Benabderrahmane, N. Bchu, P. Berteaud, M.E. Couprie, J. M. Filhol, C. Herbeaux, C. Kitegi, J. L. Marlats, K. Tavakoli, and A. Mary, in Proceedings of the 11th European Particle Accelerator Conference, Genoa, 2008 (EPS-AG, Genoa, Italy, 2008).

[5] J. Chavanne, M. Hahn, R. Kersevan, C. Kitegi, C. Penel, and F. Revol, in Proceedings of the 11th European Particle Accelerator Conference, Genoa, 2008 (Ref. [4]).

[6] S. Casalbuoni, A. Grau, M. Hagelstein, R. Rossmanith, F. Zimmermann, B. Kostka, E. Mashkina, E. Steffens, A. Bernhard, D. Wollmann, and T. Baumbach, Phys. Rev. ST Accel. Beams 10, 093202 (2007).

[7] http://ankaweb.fzk.de/.

[8] S. Casalbuoni, M. Hagelstein, B. Kostka, R. Rossmanith, M. Weisser, E. Steffens, A. Bernhard, D. Wollmann, and T. Baumbach, Phys. Rev. ST Accel. Beams 9, 010702 (2006).

[9] SHI Cryogenics Group, http://www.apdcryogenics.com.

[10] N. A. Mezentsev, in Proceedings of the 21st Particle Accelerator Conference, Knoxville, 2005 (IEEE, Piscataway, NJ, 2005).

[11] E. Wallén and G. LeBlanc, Cryogenics 44, 879 (2004).

[12] S. Casalbuoni, M. Hagelstein, B. Kostka, R. Rossmanith, E. Steffens, M. Weisser, A. Bernhard, D. Wollmann, and T. Baumbach, in Proceedings of the 10th European Particle Accelerator Conference, Edinburgh, Scotland, 2006 (Ref. [2]).

[13] The pressure values reported in this paper are measured at room temperature. The corresponding values at low temperature can be obtained applying the Knudsen relation $P(T)=(T[\mathrm{~K}] / 300 \mathrm{~K})^{1 / 2} P(300 \mathrm{~K})$.

[14] A. Kulikov, J. Seeman, and S. Heifets, in Proceedings of the 19th Particle Accelerator Conference, Chicago, Illinois, 2001 (IEEE, Piscataway, NJ, 2001).

[15] R. Cimino, A. Drago, C. Vaccarezza, M. Zobov, G. Bellodi, D. Schulte, F. Zimmermann, G. Rumolo, K. Ohmi, and M. Pivi, in Proceedings of the 21st Particle Accelerator Conference, Knoxville, 2005 (Ref. [10]).

[16] W. C. Turner, in Proceedings of the Particle Accelerator Conference, Washington, DC, 1993 (IEEE, New York, 1993).

[17] I. R. Collins and O.B. Malyshev, LHC Project Report No. 274, 2001.

[18] S. Andersson, L. Wilzén, M. Persson, and J. Harris, Phys. Rev. B 40, 8146 (1989); V. V. Anashin, G. E. Derevyankin, V. G. Dudnikov, O. B. Malyshev, V.N. Osipov, C. L. Foerster, F. M. Jacobsen, M. W. Ruckman, M. Strongin, R. Kersevan, I. L. Maslennikov, W. C. Turner, and W. A. Landford, J. Vac. Sci. Technol. 12, 1663 (1994).

[19] O. Gröbner, in Proceedings of the Particle Accelerator Conference, Vancouver, BC, Canada, 1997 (IEEE, New York, 1997).

[20] J. D. Jackson, Report No. SSC-SR-2020, 1986.

[21] LHC Design Report, http://hc.web.cern.ch/LHC/LHCDesignReport.html.

[22] V. V. Anashin, O. B. Malyshev, V.N. Osipov, I. L. Maslennikov, and W. C. Turner, J. Vac. Sci. Technol. 12, 2917 (1994).

[23] H. Tratnik, Ph.D. thesis, Wien, 2005.

[24] V. Baglin and B. Jenninger, LHC Project Report No. 721, 2004. 
[25] V. V. Anashin, O. B. Malyshev, R. Calder, O. Grbner, and A. Mathewson, Vacuum 48, 785 (1997).

[26] E. Wallén, J. Vac. Sci. Technol. 14, 2916 (1996).

[27] G. Rumolo and F. Zimmermann, Report No. CERN SLNote-2002-016.

[28] U. Iriso, S. Casalbuoni, G. Rumolo, and F. Zimmermann, in Proceedings of the Particle Accelerator Conference,
Vancouver, BC, Canada, 1997 (Ref. [19]).

[29] S. Casalbuoni, T. Baumbach, A. Grau, M. Hagelstein, R. Rossmanith, V. Baglin, B. Jenninger, R. Cimino, M. Cox, E. Mashkina, and E. Wallen, in Proceedings of the 11th European Particle Accelerator Conference, Genoa, 2008 (Ref. [4]). 